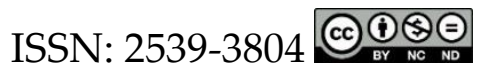

\title{
Análisis de los pronombres sujeto de primera y segunda persona del singular en cartas privadas
}

Yesenia Ramírez Fuentes Pontificia Universidad Católica de Chile

Santiago de Chile, Chile

Damian Valdés Santiago

Marlen Aurora Domínguez Hernández

Universidad de La Habana

La Habana, Cuba

\section{Resumen}

Este artículo hace un análisis lingüístico de 32 cartas originales y manuscritas, escritas en Cuba por el prócer dominicano Máximo Gómez Báez y enviadas a la poetisa puertorriqueña Lola Rodríguez de Tió entre 1900 y 1904. El objetivo de esta investigación es identificar y caracterizar los factores lingüísticos que condicionan las alternancias entre el sujeto nulo y el explícito de los pronombres de primera y segunda persona singular. Las variables lingüísticas evaluadas fueron: tiempo, modo, sintaxis y semántica verbal, cambio de referente del enunciado y énfasis. Se comprobó que la presencia de los PPSs se ve favorecida con la primera persona del singular, el modo indicativo y el tiempo pretérito. Según las variables de sintaxis y semántica verbal, los verbos transitivos, copulativos y de actividad cognoscitiva mental favorecen la presencia de los PPSs. Asimismo, el cambio de referencia resulta significativo en la explicitación de estas formas pronominales, ya que muestra a la nueva persona gramatical en la que se focalizará el enunciado. Las variables pertinentes para la ausencia de los PPSs serían, en cambio, las construcciones con formas no personales del verbo y los enunciados imperativos, ya que así lo permite el contexto.

Palabras clave: pronombres personales sujeto; primera y segunda persona singular; cartas privadas; Máximo Gómez; análisis lingüístico.

\section{Abstract \\ Analysis of first and second person singular subject pronouns in private letters \\ This paper conducts a linguistic analysis of 32 original, manuscript letters, written in Cuba by the Dominican hero Máximo Gómez Báez and sent to the Puertorican poet Lola Rodríguez de Tió from 1900 to 1904. The goal of this research is to identify and


characterize the linguistic features that conditioned the alternations between the null subject and the explicit subject of the first and second person singular pronouns. The linguistic variables considered were: time, mode, syntax and verb semantics, referent change, and emphasis. It was found that the presence of PPSs is related with the first person singular, indicative mode and past tense. According to the variables of verbal syntax and semantics, transitive, copulative and mental cognitive activity verbs foster the presence of PPSs. Likewise, the change of reference is significant in the appearance of these pronominal forms, since it shows the new grammatical person on whom the statement will be focused. The relevant variables for the absence of PPSs would be, instead, the constructions with non-personal forms of the verb and imperative statements, as the context allows.

Key words: personal subject pronouns; first and second person singular; private correspondence; Máximo Gómez; linguistic analysis.

\section{Résumé}

Analyse des pronoms personnels sujet de la première et la deuxième personne $\mathrm{du}$ singulier dans des lettres privées

Cet article présente une analyse linguistique de 32 lettres originales et manuscrites, écrites à Cuba par le héros dominicain Máximo Gómez Báez et envoyées à la poétesse portoricaine Lola Rodríguez de Tió entre 1900 et 1904. L'objectif de cette recherche est d'identifier et de caractériser les facteurs linguistiques qui conditionnent les alternances entre le sujet nul et l'explicite des pronoms de la première et de la deuxième personne en singulier. Les variables linguistiques évaluées sont : le temps, le mode, la syntaxe et la sémantique verbale, le changement de référent de l'énoncé et l'accent. Il a été constaté que la présence de PPSs est favorisée à la première personne du singulier, en mode indicatif et au passé. Selon les variables de la syntaxe et de la sémantique verbale, les verbes transitifs, copulatifs et d'activité cognitive mentale favorisent la présence de PPSs. De même, le changement de référence est significatif dans l'explicitation de ces formes pronominales, puisqu'il indique la nouvelle personne grammaticale sur laquelle l'énoncé sera axé. Les variables pertinentes pour l'absence de PPSs seraient plutôt les constructions avec des formes non personnelles du verbe et des énoncés impératifs, puisque le contexte le permet.

Mots-clés : pronoms personnels sujets ; première et deuxième personne du singulier ; lettres privées ; Máximo Gómez ; analyse linguistique. 


\section{CÓMO CITAR ESTE ARTÍCULO}

Ramírez, Y., Valdés, D., \& Domínguez, M. (2020). Análisis de los pronombres sujeto de primera y segunda persona del singular en cartas privadas. Lenguaje, 48(2), 261288. doi: $10.25100 /$ lenguaje.v48i2.8486 


\section{INTRODUCCIÓN}

Ante la imposibilidad de contar con repertorios del coloquio oral que resulten ilustrativos de las formas de habla de los siglos precedentes, las fuentes escritas se han convertido en un material ineludible para la realización de trabajos desde los enfoques de la lingüística histórica. Tener acceso a textos de diferentes épocas permite establecer un estado comparativo de las formas, que podría inducir al investigador a elaborar una posible genealogía del cambio lingüístico, además de poder encontrar un grupo de generalidades que permitirían anclar un texto a una época, estilo o autor, a partir de las peculiaridades lingüísticas discursivas que se evidencien en el examen del documento.

Según las personas que intervienen en su elaboración, la época en que fueron escritos, sus contenidos y funciones, podemos hablar de textos jurídicos, administrativos, literarios o privados. Estos últimos han sido privilegiados en los estudios lingüísticos por la creencia de que, en alguna medida, se acercan más a las peculiaridades de la oralidad de cada época y, por lo tanto, ofrecerán más pistas de lo que ocurre en esa habla real. $\mathrm{Y}$ en este punto adquieren un valor especial las cartas personales escritas a familiares o amigos y elaboradas por personas de bajo nivel educacional, circunstancias ideales que propiciarían la neutralización de algunas de las presiones de la lengua escrita a favor de la espontaneidad. Aunque estos sean elementos que se arguyen para fundamentar la idoneidad de esta tipología documental, no debe ignorarse la importancia de la que también gozan los otros tipos textuales; ya que todos, de cierta forma, reúnen una condición que los homologa: su valor testimonial. La investigación que presentamos pretende dar continuidad a los análisis lingüísticohistóricos de documentos privados y a los esfuerzos que se han hecho en la descripción y caracterización del español de América, y de forma más circunscrita de la variedad antillana de la lengua.

Nuestra investigación se nutre de todos los trabajos que hemos referido y se inserta en la tarea de la descripción y caracterización de la lengua, pero con sus propios derroteros. Es por ello que hemos tomado como corpus 32 cartas elaboradas (8021 palabras) en Cuba por el prócer dominicano Máximo Gómez Báez y enviadas a la poetisa puertorriqueña Lola Rodríguez de Tió. Será muy favorecedora la homogeneidad de este corpus, pues se trata de un solo destinador y un único destinatario de los cuales se tienen abundantes datos (edad, origen social, lugares de nacimiento y residencia, ideario político, etc.), y que podrían favorecer el análisis del geolecto antillano. Esta homogeneidad también podría derivar en una de las limitaciones de la investigación, ya que contar con cartas cruzadas sería lo óptimo en una investigación de este tipo ${ }^{1}$.

\footnotetext{
${ }^{1}$ En la revisión documental realizada hasta el momento, solo se han encontrado en el Archivo Nacional de Cuba dos cartas originales enviadas por Lola a Gómez en el periodo objeto de estudio (1900-1904), cantidad que no resultó representativa para incluirla en el presente análisis.
} 
El período en el que se enmarca esta correspondencia abarca los años comprendidos entre 1900 y 1904, cuya norma de referencia está asentada en el español de la América decimonónica y en las regulaciones de la Real Academia Española para esta época. Se suma a estas coordenadas el hecho de que los textos analizados son cartas originales manuscritas, lo que avala la fiabilidad de las conclusiones lingüísticas. Una tarea indispensable para el estudio de la selección documental fue el trabajo editorial que consistió en la transcripción de cada una de las misivas (cfr. Anexo 1).

A partir de un estudio esencialmente filológico nos proponemos como objetivos de la investigación identificar y comentar las variables lingüísticas con las cuales se relaciona la presencia/ausencia de los pronombres personales sujeto de primera y segunda persona del singular. Las conclusiones que se deriven de este trabajo pueden constituir un aporte para los estudios de la variedad antillana del español en el siglo XIX.

\section{El hallazgo}

La Lic. Ariamna Alonso Serra, responsable de la biblioteca de la Academia Cubana de la Lengua, localizó, entre sus fondos más antiguos, 32 cartas originales manuscritas elaboradas en Cuba (específicamente en el poblado de Calabazar de La Habana) por el prócer dominicano-cubano Máximo Gómez Báez y enviadas a la poetisa puertorriqueña Lola Rodríguez de Tió en el período 1900-1904. Por la importancia de ambas figuras, se consideró pertinente fotocopiar y transcribir las cartas para su mejor conservación y estudio.

\section{MARCO TEÓRICO-METODOLÓGICO DE LA INVESTIGACIÓN. FUNDAMENTACIÓN DEL ANÁLISIS LINGÜÍSTICO}

Pese al interés que el español del Caribe (antillano) ofrece para la dialectología hispanoamericana, esta variedad todavía espera por más estudios que contribuyan a la descripción de su desarrollo. Los nuevos enfoques abogan por trabajos sistemáticos que tomen como base los principios de la dialectología histórica y el análisis de documentos de acuerdo con su tipología textual. La revisión de las investigaciones sobre el español del área nos permite advertir que no ha prevalecido entre los autores la preocupación por el aprovechamiento de los documentos de carácter personal para realizar trabajos de corte lingüístico histórico. Precisamente en esta línea, el presente estudio pretende ser una contribución a la historia lingüística de la región.

Entre las características consensuadas definitorias del español caribeño se encontraba la significativa presencia de sujetos pronominales en el español de esta zona dialectal (Cameron, 1993; Hurtado, 2005; Ortiz, 2009a; López, 1992; Morales, 1999; Otheguy y Zentella, 2007; Vandermeulen, 2011), incluso desde las primeras 
descripciones de la variedad caribeña de la lengua (López, 1999), por lo que algunos de los estudiosos lo señalan como un rasgo dialectal de la zona (Méndez y Moreno, 2014; Pérez, 2006; Sánchez, 2013).

De los textos examinados y cotejados sobre este tema se colige que el número singular suscita la explicitación de los pronombres personales sujetos (Abreu, 2009, 2012; Bentivoglio, 1987; Claes, 2011; Flores, 2004, 2009) y que son los de primera y segunda persona singular los de mayor frecuencia (Claes, 2011; Enríquez, 1984; Morales, 1999).

Estos criterios motivaron el estudio de los factores lingüísticos que condicionan las alternancias entre el sujeto nulo y el explícito de los pronombres de primera y segunda persona singular (PPS) en los documentos que se enmarcan, justamente, en el área dialectal caribeña hispánica insular.

La presencia de los PPSs en función sujeto en español no es necesaria la mayoría de las veces, condición que está asociada a la riqueza morfológica del paradigma verbal (Fernández, 1999; Orozco y Guy, 2008; Prieto, 2011; Rouges, 2011). Este carácter sintético de las formas verbales contribuye a la libertad constructiva de nuestro idioma y contrasta con otras lenguas (como el inglés o el francés) en las que la anteposición del sujeto pronominal es obligatoria.

Si la presencia de los PPSs en función sujeto en español no es necesaria: ¿por qué se produce su explicitación? El objetivo de este trabajo es identificar y caracterizar factores lingüísticos que condicionan las alternancias entre el sujeto nulo y el explícito de los PPSs en este corpus textual, que se enmarca en el área dialectal caribeña hispánica insular.

Se destacan en este sentido los factores lingüísticos que pueden condicionar la variación de los PPSs, según las investigaciones de diversos autores como Bentivoglio (1987), Cameron (1993), Flores (2004), Hurtado (2005), Méndez y Moreno (2014). Morales (1999), Orozco y Guy (2008), Prieto (2011), Rouges (2011), Sánchez (2013), Silva (1982), Vandermeulen (2011) y Vidal (2013). Estos factores pueden ser de carácter gramatical como la ambigüedad morfológica, la persona y número gramatical, de tipo pragmáticodiscursivo como el cambio de referencia, y de origen semántico como la clase de verbo, entre otros ${ }^{2}$.

Para el presente estudio nos hemos basado en el esquema de trabajo que propone Prieto (2011), ya que permite correlacionar la presencia y la ausencia de los pronombres personales en función sujeto en diferentes contextos, según las siguientes variables: tiempo, modo, sintaxis y semántica verbal, y cambio de referente del enunciado. Otro de los aspectos que se dice influyen en la presencia/ausencia de los PPSs es el énfasis. Es por ello que esta categoría también fue evaluada en nuestros documentos. La propuesta de

\footnotetext{
${ }^{2}$ Vaquero (1996) añade a estos factores la tendencia al orden sujeto-objeto-verbo.
} 
Prieto (2011) no se trasladó tal cual a nuestro estudio pues se le hicieron algunos ajustes que se irán precisando en el desarrollo de cada una de estas variables.

\section{PPSs según el tiempo y modo verbal}

El tiempo y el modo verbal son las categorías lingüísticas presentes de modo más sistemático en el análisis de la forma explícita y nula de los PPSs, porque afectan a toda la oración, como lo prueba su consideración en los autores que hemos ido mencionando.

Se conoce que los paradigmas verbales difieren respecto a la información desambiguadora relacionada con el sujeto, lo que podría arrojar luces sobre el comportamiento de los PPSs. El empleo de estas formas podría estar favorecido por el hecho de que no esté suficientemente determinada por el contexto la persona o cosa a que nos referimos, lo que supone por parte del que habla el deseo de eliminar una falsa interpretación posible. Por lo tanto, las conjugaciones verbales en las que las personas gramaticales sean equivalentes $\mathrm{y}$, por lo tanto, no distintivas podrían ser útiles para evaluar hasta qué punto esta variable podría justificar los usos explícitos.

La conclusión de Orozco y Guy (2008) en este aspecto es sumamente provocadora pues señalan que no existen diferencias apreciables entre los paradigmas más distintivos y los menos distintivos, ya que ambos favorecen igualmente la aparición de sujetos explícitos. Los estudios de Prieto (2011) revelan, en cambio, que sí existen diferencias significativas entre los PPSs presentes y ausentes, según el tiempo verbal. Ambas conclusiones invitan a estudiar a fondo los efectos de esta variable.

El modo verbal corre con menor fortuna en el rango de importancia en el análisis del comportamiento de los PPSs. Para Prieto (2011) este elemento ofrece escaso interés, pues la prueba estadística que realizó reveló que no había diferencias significativas cuando se correlacionan los PPSs presentes y ausentes con el modo verbal.

\section{PPSs según la sintaxis y semántica verbal}

La sintaxis verbal se refiere a la relación entre el alcance del verbo en la cláusula y la presencia/ausencia de los PPSs. En este sentido, Prieto (2011) valora como posible indicador el carácter transitivo, intransitivo o copulativo de los verbos. La transitividad del verbo desempeña un papel importante en el uso explícito del pronombre personal porque cambia el foco de atención (Posio, 2011; Rouges, 2011). Las construcciones copulativas también pueden ser interesantes para valorar el comportamiento de los PPSs, ya que los verbos funcionan como nexos entre el sujeto y el atributo.

La variable semántica también se integra al análisis con el fin de examinar las cualidades temporales propias de la situación designada por un verbo (Bentivoglio, 1987; Enríquez, 1984; Flores, 2004, 2005, 2007; Hurtado, 2005; Orozco y Guy, 2008; Prieto, 2011; Rouges, 2011; Silva, 1982). 
Para este factor no parece que haya una tendencia clara pues en los estudios donde se ha tomado en consideración este parámetro coexisten algunas divergencias. Así por ejemplo, Bentivoglio (1987) y Hurtado (2005) consideran que un pronombre personal sujeto tiene probabilidades de ser expresado con verbos de cognición (Tú conoces todos las posibles soluciones, Yo investigué sobre lo que hablamos).

Por otra parte, según los resultados de Enríquez (1984), los verbos estimativos (Yo me imagino cuando me lo describes) tienen un alto grado de subjetividad, por lo que favorecen la presencia del pronombre personal sujeto (54\%). Para este autor, los verbos estimativos son aquellos que expresan opiniones o juicios de valor e incluyen, entre otros: creer, considerar, imaginar, pensar, opinar, etc.

La mayor dificultad en este apartado tiene que ver con la arbitrariedad de los conceptos que se manejan en la clasificación semántica de los verbos y que se verifica en el repertorio de términos para describir el mismo tipo de verbo: verbos de percepción intelectual (Cano, 1981), verbos de conocimiento (Comesaña, 2002), verbos de actitud proposicional (De Saeger, 2006; Shinzato, 2004), verbos de cognición (Kervorkian y Pacagnini, 2010), verbos de actividad mental (Martínez, 2010) y verbos de representación mental (Kervorkian y Pacagnini, 2010). A esto sumémosle el carácter polisémico de algunos verbos, lo que dificulta todavía más una clasificación estable.

Lejos de ser un ámbito de estudio con unos principios teóricos y metodológicos consensuados, la bibliografía sobre la semántica verbal se caracteriza por la multiplicidad de propuestas de clasificación, que no solo difieren en cuanto al número y la organización de las clases aspectuales, sino también en el nivel lingüístico en el cual se manifiestan dichas clases. Prieto (2011) propone la dualidad: verbos estativos/dinámicos.

Se prefiere para el presente estudio una clasificación más descriptiva y matizada con el fin de ser más precisos en tanto se valoren más posibilidades. En este sentido nos parece más apropiada la que ofrece Bentivoglio (1987) que clasifica los verbos de la siguiente forma: verbos de actividad cognoscitiva y mental (creer, decidir, imaginar, asumir, estar de acuerdo, asegurar, pensar, olvidar, recordar, saber, asegurar), verbos de percepción y sensación (oler, oír, sentir, ver), verbos de dicción o pronunciación oral (afirmar, comentar, decir, sugerir) y verbos de volición (pretender, querer, desear). A estas cuatro categorías hemos añadido la de los verbos estativos (ser, estar, tener) y dinámicos (ir, comer), por la importancia que merecen estos verbos en los trabajos de Villegas (2006) y Prieto (2011). Varios autores coinciden en que los verbos de actividad mental y de comunicación son los que presentan mayor tendencia a aparecer con sujetos pronominales explícitos (Bentivoglio, 1987; Morales, 1997; Villegas, 2006). Esto se debe a que estos verbos presentan una carga importante de subjetividad, ya que expresan acciones propias de los seres humanos relacionadas con la manifestación de una opinión o comentario, y todo ello parece crear contextos propicios para el refuerzo del sujeto (Morales, 1997). 


\section{PPSs según la información nueva o conocida. El cambio de referencia}

Los estudiosos consideran que el cambio de referencia (información nueva "rema" o conocida "tema") repercute en la información explícita o tácita de los PPSs. Algunos de los trabajos que se han referido a este factor son los de Bentivoglio (1987), Cameron (1993), Flores (2004), Méndez y Moreno (2014), Orozco y Guy, (2008), Ortiz (1999), Prieto (2011), Rouges (2011) y Silva (1982). Ortiz (1999) afirma que ante una misma referencia, los hablantes se inclinan por la no explicitación del pronombre, mientras que ante un cambio de referente apoyan la presencia pronominal, por lo que el cambio de referente favorecería el uso de los sujetos explícitos. Los estudios de esta variable se han enfocado no solo en el cambio de referente sujeto inmediato anterior, sino también en cambios que se consideran parciales como la correferencialidad con el objeto directo o indirecto (Orozco y Guy, 2008).

\section{PPSs según el énfasis}

Ya hemos mencionado que el español admite la posibilidad de omitir el sujeto y que, a pesar de eso, la variante caribeña presenta altos índices de PPSs expresos. Este elevado empleo ha sido justificado, además de por los factores ya antes trabajados, por la intención del hablante de enfatizar la participación del sujeto en la acción.

Las gramáticas tradicionales coinciden en que el uso de los sujetos pronominales resulta, en su mayoría, redundante. Esto se debe a la capacidad del español de incluir la información de persona en la desinencia verbal. Gili Gaya (1975) afirma que los pronombres de primera y segunda persona, usados explícitamente, siempre resultan enfáticos. Por su parte, el Esbozo de una nueva gramática de la lengua española (Real Academia de la Lengua Española, 2006) plantea que solo dos casos admiten la presencia explícita del pronombre sujeto: el uso enfático y el desambiguador, ante las desinencias homólogas de primera y tercera persona. A este pensamiento se unen Enríquez (1984), Llorach (1994) y Luján (1999).

La explicación tradicional resulta a todas luces deficiente para la descripción de este fenómeno en el español caribeño. Investigaciones más recientes han aportado nuevos datos y perspectivas sobre el uso explícito de los pronombres y la categoría énfasis, sin llegar por ello a ser suficientes (Pérez, 2006; Rouges, 2011; Vandermeulen, 2011).

En cuanto al uso desambiguador de estos pronombres, Pérez (2006) afirma que la homonimia desinencial puede darse también por el uso de la forma de segunda persona en una estructura como ¿qué tú dice?. Este fenómeno específico ha sido trabajado, además, por Hochberg (1986) y Vandermeulen (2011) para el español puertorriqueño. La descompensación fonológica, dada por la pérdida de la /-s/ en las desinencias verbales, 
es solventada por la explicitación del pronombre, lo que permite desambiguar las formas fonológicamente iguales. Por tanto, este uso resultaría no enfático.

Estas investigaciones han relacionado el uso enfático de los pronombres sujeto con contextos específicos. Vandermeulen (2011) afirma que algunos predicados presentan marcas contrastivas, mediante el uso de las conjunciones pero, más y sin embargo, y de refuerzo, cuando el sujeto es seguido, entre otros, del adjetivo mismo/a o el adverbio justamente. Pérez (2006) menciona, además, los casos en que el sujeto va precedido de hasta, incluso o excepto y seguido del adverbio sí. Rouges (2011) añade la anteposición del adverbio tampoco y la introducción de nueva información.

Se han considerado, también, como usos enfáticos del pronombre las estructuras de reduplicación (María quiere hacerlo ella) y algunas oraciones subordinadas (como tú quieras) (Fernández, 1999). Sin embargo, hay ejemplos en los que, por el contexto, no puede emitirse una clara valoración de los usos enfáticos. En estos casos, la clasificación dependerá entonces de la interpretación personal del investigador.

\section{Resultados}

En el corpus se encuentran un total de 446 verbos con posibilidad de un pronombre de primera o segunda persona singular presente o ausente (Tabla 1). De las relaciones estadísticas presentadas se desprende que los PPSs ausentes representan el $72.4 \%$, lo que confirma que se sigue el parámetro de sujeto tácito que se supone caracteriza al español estándar (Fernández, 1999; Orozco y Guy, 2008; Prieto, 2011; Vandermeulen, 2011).

Tabla 1. Ausencia y presencia de los PPSs

\begin{tabular}{|l|l|l|l|l|l|}
\hline \multirow{2}{*}{ Presentes } & \multirow{2}{*}{123} & \multirow{2}{*}{$27.6 \%$} & yo & 94 & $76.4 \%$ \\
\cline { 4 - 6 } & & & tú & 29 & $23.6 \%$ \\
\hline \hline \multirow{2}{*}{ Ausentes } & \multirow{2}{*}{323} & \multirow{2}{*}{$72.4 \%$} & $\mathbf{1}^{\text {ra }}$ & 226 & $70.0 \%$ \\
\cline { 4 - 6 } & & & $\mathbf{2}^{\text {da }}$ & 97 & $30.0 \%$ \\
\hline
\end{tabular}

Se destaca, además, en el corpus, la alta frecuencia, entre los sujetos expresos, de la primera persona del singular $(76.4 \%)$ sobre la segunda $(23.6 \%)$, un resultado que concuerda con las conclusiones de investigaciones anteriores (Enríquez, 1984; Orozco y Guy, 2008; Vandermeulen, 2011). 


\section{PPSs según el tiempo y modo verbal}

En el análisis se tomaron en cuenta todos los tiempos utilizados con verbos pertenecientes a la primera y segunda persona del singular ${ }^{3}, \mathrm{y}$ fueron agrupados en los modelos temporales verbales de la Tabla 2.

Tabla 2. Presencia/ausencia de los PPSs según el tiempo verbal

\begin{tabular}{|l|l|l|l|l||l|}
\hline \multirow{2}{*}{ Tiempos verbales } & \multicolumn{2}{|l|}{ Presentes } & \multicolumn{2}{l||}{$\begin{array}{l}\text { Ausentes } \\
\text { (Cant.) }\end{array}$} \\
\cline { 2 - 5 } & Cant. & $\%$ & Cant. & $\%$ & (a) \\
\hline \hline Presente & 101 & $29.8 \%$ & 238 & $70.2 \%$ & 339 \\
\hline Pretérito & 19 & $29.2 \%$ & 46 & $70.8 \%$ & 65 \\
\hline Futuro & 2 & $10.0 \%$ & 18 & $90.0 \%$ & 20 \\
\hline
\end{tabular}

La información más pormenorizada de las frecuencias asignadas a cada tiempo verbal se encuentra en la Tabla 3:

Tabla 3. Presencia/ausencia de los PPSs según el tiempo verbal

\begin{tabular}{|c|c|c|c|c||c|}
\hline \multirow{2}{*}{ Tiempos verbales } & \multicolumn{2}{|c|}{ Presentes } & \multicolumn{2}{c||}{ Ausentes } & Total \\
\cline { 2 - 5 } & Cant. & $\%$ & Cant. & $\%$ & (Cant.) \\
\hline \hline Presente & 82 & $29.0 \%$ & 201 & $71.0 \%$ & 283 \\
\hline Antepresente & 19 & $34.0 \%$ & 37 & $66.0 \%$ & 56 \\
\hline Pretérito & 9 & $25.0 \%$ & 36 & $75.0 \%$ & 45 \\
\hline Futuro & 2 & $10.0 \%$ & 18 & $90.0 \%$ & 20 \\
\hline Copretérito & 8 & $57.0 \%$ & 6 & $43.0 \%$ & 14 \\
\hline Antecopretérito & 1 & $25.0 \%$ & 3 & $75.0 \%$ & 4 \\
\hline Pospretérito & 1 & $100 \%$ & - & - & 1 \\
\hline Antepretérito & - & - & 1 & $100 \%$ & 1 \\
\hline
\end{tabular}

El tiempo verbal más empleado en el corpus es el presente (283), seguido por el antepresente (56) aunque con una notable diferencia entre ellos. Las incidencias del antecopretérito, pospretérito y antepretérito representan, en nuestros documentos, menos del $1 \%$ del total de verbos conjugados (424), por lo que no se consideraron significativas para el análisis.

3 Estos son: presente, pretérito, futuro, copretérito, pospretérito, antepresente, antepretérito y antecopretérito. Por razones de factibilidad estadística y en aras de reducir las tablas de contingencias, se decidió agrupar estos tiempos en los tres modelos verbales prototípicos: presente, pretérito y futuro (Tabla 2). La información más pormenorizada de las frecuencias asignadas a cada tiempo verbal se encuentra en la Tabla 3. 
Al realizar la prueba ji-cuadrado ${ }^{4}$ se confirmó que hay una asociación estadísticamente significativa entre el tipo de tiempo verbal y la presencia o ausencia de los PPSs en el corpus analizado $\left(X^{2}=14.26, g l=7, p=0.04\right)$.

Se comprueba que la presencia de los pronombres personales sujetos está favorecida por el empleo del tiempo copretérito y también puede observarse que es el único tiempo en el que existen más incidencias de pronombres presentes que ausentes. Esta influencia del tiempo copretérito en la presencia de los PPSs puede explicarse por la homomorfia de las formas verbales de primera y tercera personas, y acaso de la segunda, en este tiempo ${ }^{5}$, por lo que su empleo tendría una clara función desambiguadora (Fernández, 1999; Hurtado, 2005; Orozco y Guy, 2008; Rouges, 2011; Vandermeulen, 2011).

Esta idea de la desambiguación se confirma en algunos ejemplos de los documentos en los que la presencia de los pronombres sujetos aclara falsas interpretaciones posibles:

Santacilia ha juzgado bastante bien á Manuel Sanguily si le pudieramos quitar un poco de apasionamiento yo pensaria en él para Presidente de la Republica. ${ }^{6}$

Estimada y complaciente amiga. Ya sabia yo que en la bondad de tu corazon y tu cararter no cabia la negativa á mi invitacion para la velada en Santiago de las Vegas.

Por mi parte lo pasé como yo deseaba, solo con mis pensamientos.

Y oye bien lo que decia yo al calor de mi hogar, leyendo tu carta como si leyera un troso de la vida de Santa Teresa de Jesus.

La mayoría de los estudios revelan que la alta frecuencia de pronombres personales sujetos en el contexto caribeño se relaciona con la ambigüedad morfológica derivada de la omisión de la /-s/. Este fenómeno provoca una indistinción entre la segunda y la tercera persona singular, por lo que motivaría la expresión del sujeto para compensar la pérdida de este fonema en la desinencia verbal y desambiguar la expresión (Flores, 2004; Méndez y Moreno, 2014; Rouges, 2011; Sánchez, 2013; Vandermeulen, 2011). No documentamos en nuestras cartas la elisión de este fonema en las desinencias verbales. Al respecto véanse los siguientes ejemplos:

\footnotetext{
${ }^{4}$ Se utilizó el software SPSS (Statistical Package for the Social Sciences) para el cálculo de la prueba jicuadrado con los datos de las Tablas 2 y 3.

${ }^{5} \mathrm{La}$ ambigüedad morfológica entre la primera y la tercera persona singular también se verifica para los tiempos Pospretérito, Antecopretérito y Antepospretérito del modo indicativo y en todos los tiempos del modo subjuntivo (Tabla 3).

${ }^{6}$ En los ejemplos que se presentan en este trabajo se respeta la ortografía original de las misivas.
} 
Pues aunque ella quiera y tu la desees ya Patria es agena...

Ya hablarémos de todo éso cuando tu termines de abrasar á tus numerosos amigos.

Las amigas a las que saludas por mi conducto te retornan sus cariños.

Por eso le has puesto ése nombre al ser que mas ámas...

Los datos correspondientes a la presencia/ausencia de los pronombres personales sujeto de primera y segunda persona singular con respecto a la variable modo verbal se despliegan en la Tabla 4:

Tabla 4. Presencia/ausencia según el modo verbal

\begin{tabular}{|c|c|c|c|c||c|}
\hline \multirow{2}{*}{ Modos verbales } & \multicolumn{2}{|c|}{ Presentes } & \multicolumn{2}{c||}{ Ausentes } & Total \\
\cline { 2 - 5 } & Cant. & $\%$ & Cant. & $\%$ & (Cant.) \\
\hline \hline Indicativo & 115 & $31.5 \%$ & 250 & $68.5 \%$ & 365 \\
\hline Subjuntivo & 7 & $22.6 \%$ & 24 & $77.4 \%$ & 31 \\
\hline Imperativo & - & - & 28 & $100 \%$ & 28 \\
\hline
\end{tabular}

El modo verbal más utilizado es el indicativo, en segundo lugar, estaría el subjuntivo y por último el imperativo. Esta descripción está acorde con las características de los documentos, pues son cartas en las que el autor narra, comenta y manifiesta ideas, pensamientos y creencias. La actitud del hablante frente al enunciado rara vez es de posibilidad, expectativa o contingencia; más bien los hechos se perciben por el destinador como reales y certeros, por lo tanto, merecedor de ser comentados con otra persona, aunque, en algunas ocasiones, también exhorta o solicita a su interlocutora que le brinde su opinión al respecto.

Se comprueba en nuestros documentos que el empleo de los pronombres de primera y segunda persona singular se ve favorecido por el modo indicativo. Resulta igualmente interesante la tendencia generalizada del sujeto pronominal tácito de segunda persona en el modo imperativo. Este comportamiento es absolutamente comprensible pues la modalidad enunciativa que responde a la estructura imperativa (por su función apelativa en el discurso) siempre se dirigirá a la segunda persona singular o plural, por lo que no resultaría necesaria la presencia de estas formas pronominales, pues no se genera ninguna ambigüedad en la interpretación.

Al realizar la prueba ji-cuadrado, se confirmó que hay una asociación estadísticamente significativa entre el modo verbal y el comportamiento variable de los PPSs $\left(X^{2}=13.22, g l=2, p=0.001\right)$. 


\section{PPSs según la sintaxis y semántica verbal}

Rouges (2011) señala que el énfasis depende del nivel de transitividad, por lo que esta categoría sintáctica del verbo sería determinante en el uso explícito del pronombre personal. En una oración transitiva el objeto se encuentra afectado por la acción, por lo que es un foco de atención, de lo que se deriva que el objeto directo tendría más protagonismo que el sujeto. En consecuencia, la frecuencia de los pronombres personales sujetos en la oración transitiva sería menor, pues otro elemento de la oración merecerá mayor connotación. Lo contrario ocurrirá, entonces, con las oraciones intransitivas.

Contrario a lo que plantea Rouges, nuestros documentos reflejan una mayor tendencia a la presencia de los PPSs en cláusulas transitivas. Prieto (2011) concluye en el análisis de esta variable que la mayoría de los PPSs son utilizados con los verbos transitivos $(78 \%)$, criterio que se confirma en el análisis de nuestras cartas.

Yo creo que hemos salvado la Patria del naufragio, pero nosotros hemos quedado mas que fatigados, extenuados.

Yo sé que tu ideal es la Patria...

Tu no sabes como me traen ahora a mi los mismos cubanos.

Ya tu conoces muy bien la puntualidad de la raza latina...

Resulta importante señalar la alta frecuencia de PPSs expresos en construcciones copulativas:

Terminada mi mision el dia 20 debo ya ocuparme del problema de los mios, y no digo de mí pues ya yo soy viejo con rumbo á la fosa y muy poco es lo qué necesito.

Yo no soy mas que un hombre qe ama á Cuba y que no puede ser feliz si ella no lo es.

Ella quizas no lo sabe, pero es muy posible que lo haya oido contar atí, "que yo soy hombre de una sola palabra"

Yo no soy hombre de mentiritas, y si es verdad que estúbe el Domingo en la Habana no quise ir á verte sin poderte [interlineado: dar] una noticia agradable $p^{a}$ que mi visita lo fuera tambien_

Los verbos de actividad cognoscitiva y mental (creer, saber, pensar, imaginar, olvidar, recordar) representan la frecuencia más alta con respecto a las otras clasificaciones y propician de manera significativa la explicitación de los PPSs. En 
ocasiones, algunos de estos verbos están acompañados de complementos directos, por lo que estos dos factores (semántica y sintaxis verbal) vienen a ser significativos para valorar el comportamiento de los PPSs:

Si tu no te esplicas tus tristezas, profundisa un poco y en esas dudas encontraras la Causa.

Yo conosco mas que ninguno de sus amigos el cararter de ése hombre.

Yo creo que tu como David con su arpa tienes el poder de amansar las fieras.

Yo que creo que sin amor la vida es un infierno estoy contentisimo cuando noto que los cubanos unos á otros se respetan pues me queda á mi la esperanza de qué algundia se me quiera y respete también.

El hecho de que sean estos verbos los más frecuentes en los textos, se corresponde con las ideas que hemos estado desarrollando a lo largo del trabajo. Al emisor le interesa que su interlocutora conozca todo lo que él piensa con respecto a lo que le ocurre, por lo que su discurso se organiza de manera expositiva argumentativa.

Los verbos de dicción o pronunciación (afirmar, comentar, decir, sugerir) constituyen el segundo grupo de interés y en tercer lugar los verbos de percepción (oír, ver, sentir):

Cuando yo dije éso á Gilberto el Ministro, se quedó admirado, y á mi me causó pena, pues yo me dije, "éste hombre no me creia capaz de sentir y ver".

Por eso tu tambien sientes "ese fondo de tristeza intima, inexplicable á veces en todos los corazones que saben sentir".

De manera que, siguiendo la propuesta que hace Prieto (2011) para la semántica verbal con la clasificación de estativos o dinámicos, la presencia de los PPSs se ve favorecida en nuestros textos con los verbos estativos, conclusión que se corresponde con los resultados de la investigación de dicha autora: la mayoría de los PPSs son utilizados con los verbos estativos (79\%).

Esta clasificación refiere que el verbo estativo sería aquel en el que persiste un único estado, es decir, que no cambia (ser, tener, amar, saber, esperar, mirar, pensar) y los dinámicos serían aquellos en los que se denota un cambio o progreso en el tiempo (bailar, caminar, correr, decir, huir, ir), por lo que el parámetro que distingue los predicados dinámicos de los predicados estativos es el parámetro del cambio (Lyons, 1971; Prieto, 2011). 


\section{PPSs según la información nueva o conocida. El cambio de referencia}

Silva (1996) en su investigación basada en entrevistas orales grabadas del español de méxico-americanos de Los Ángeles, pretende probar que la relativa libertad de la posición del sujeto en español responde a factores pragmáticos del tipo cambio de referente. Propone que un cambio de referente se da cuando el sujeto tiene un referente distinto al del sujeto de la oración anterior (como en la frase A). Sin embargo, cuando se mantiene el mismo referente (como en la frase B) solo se expresa explícitamente el sujeto en solo una de las oraciones.

\section{A. Él era hijo único. Yo soy hijo único también.}

B. Yo siento que Ø tengo que cuidar.

Ortiz (2009b) en su estudio sobre los pronombres en el español del Caribe como primera y como segunda lenguas, anota que ante una misma referencia (cfr. B), los hablantes se inclinan por la referencia nula, mientras que ante un cambio de referente (cfr. A) estos mismos hablantes apoyan la presencia pronominal.

En nuestros documentos se corrobora que el cambio de referencia puede ser una de las variables que motiva la presencia de los PPSs:

Pues aunque Ella quiera y $\underline{\text { tu }}$ la desees, ya Patria es agena y ésto como dice el refran de servir á dos amos es cosa que no sale muy derecho.

Y yo te soy franco (tu sabes que no soy un bobo).

Como has hecho tu ahora, y he hecho yo hace mucho tiempo.

\section{Énfasis}

Algunos autores señalan al énfasis como una de las variables que propician la aparición del sujeto pronominal, entre ellos se encuentran: Bentivoglio (1987), Enríquez (1984), Fernández (1999), Orozco y Guy (2008), Rouges (2011) y Vandermeulen (2011). La descripción exacta de esta influencia no ha ido más allá del planteamiento de algunas estructuras o combinaciones consideradas enfáticas (Fernández, 1999; Pérez, 2006; Rouges, 2011; Vandermeulen, 2011). A pesar de lo dicho anteriormente, no siempre queda claro cuándo un sujeto pronominal tiene valor enfático o no, lo que nos da la medida de lo complejo del asunto.

En esta primera aproximación se asociarán las estructuras señaladas como favorecedoras del énfasis, según las investigaciones consultadas, con los casos que se correspondan en nuestro corpus, y se agruparán y comentarán aquellos ejemplos que no se insertan en dichas estructuras sin pretender con ello arribar a conclusiones categóricas. 
El corpus contiene 123 PPSs expresos y la mayoría (76.4\%) corresponde a pronombres de primera persona. La Tabla 5 muestra el balance de los pronombres personales sujetos de primera y segunda persona singular presentes:

Tabla 5. PPSs presentes

\begin{tabular}{|l|l|l|l|l|l|}
\hline \multirow{2}{*}{ Presentes } & \multirow{2}{*}{123} & \multirow{2}{*}{$27.6 \%$} & yo & 94 & $76.4 \%$ \\
\cline { 4 - 6 } & & & tú & 29 & $23.6 \%$ \\
\hline
\end{tabular}

La ambigüedad morfológica ha sido considerada por las gramáticas tradicionales, junto con el énfasis, como una variable que justifica la aparición del pronombre. Podría clasificarse entonces esta necesaria mención del sujeto pronominal, debido a la ambigüedad morfológica, como un uso no enfático.

A continuación, presentamos el listado de pronombres explícitos en contextos de ambigüedad morfológica:

Por mi parte lo pasé como yo deseaba, solo con mis pensamientos.

A la vez que el tiempo ha estado muy malo, yo no me he sentido bien de salud, por éso no he ido á abrasarte y á verte ése ojo que, ahora te lo digo, yo creia sin luz (...)

Ya sabia yo que en la bondad de tu corazon y tu cararter no cabia la negativa á mi invitacion para la velada en Santiago de las Vegas.

Si le pudieramos quitar un poco de apasionamiento, yo pensaria [interlineado: en] él pa Presidente de la Republica.

En estos ejemplos la ambigüedad se da con un caso para el pretérito de subjuntivo y para el modo indicativo, con siete ejemplos del tiempo copretérito y uno del pospretérito.

Las construcciones con formas no personales del verbo no son capaces por sí solas de otorgar información de persona gramatical por lo que algunos autores consideran que estas estructuras favorecen la presencia de sujetos pronominales (Sarmiento y Esparza, 1994; Suñer, 1986; Vandermeulen, 2011), circunstancia que podría llevarnos a pensar en un uso no enfático, por la necesidad de señalar la información de persona mediante el uso expreso del pronombre.

Si puedes venir me lo dises para yo preparar, no la venida, que esa es fácil, sino el regreso que es lo penoso.

Otro de los contextos que ha sido descrito en la bibliografía como favorecedor de los PPSs enfáticos es el uso antepuesto de las conjunciones pero, mas, sin embargo y aunque 
a las formas pronominales, lo que supondría un énfasis por contraste (Rouges, 2011; Vandermeulen, 2011). Nuestro corpus presenta algunos ejemplos de estos usos:

Una comision vino á invitarme para que lo Presidiera, pero yo me escuse como pude (...)

Si alguna vez he sentido no ser poeta ha sido ahora para en versos tambien, decirte todos los agradecimientos de mi corazon. Pero tu [interlineado: me] consideras sincéro qe es igual á la lealtad.

Aquellos eran otros tiempos, la bestia humana relinchiba por todas partes, y asi tenia que suceder y el Mundo soportar esa desgracia que no otra cosa son las guerras pero despues de la Paz, yo todo lo he perdonado, y no quisiera que se hablara de cosas de la guerra.

El uso posverbal del pronombre sujeto de segunda persona de respeto (usted) no se considera enfático, mientras que cualquier otro pronombre en esta posición puede considerarse como altamente contrastivo (Fernández, 1999; Rouges, 2011). La muestra contiene algunos ejemplos de PPSs en posición posverbal:

Estimada y complaciente amiga. Ya sabia yo que en la bondad de tu corazon y tu cararter no cabia la negativa á mi invitacion para la velada en Santiago de las Vegas.

Y oye bien lo que decia yo al calor de mi hogar, leyendo tu carta como si leyera un troso de la vida de Santa Teresa de Jesus.

(...) por un lado no me gustan ésos banqueteos celebrando cosas sagradas del ideal de la independencia, y no saboreo yo la independencia.

Ya sé yo que Lily te quiere pues ella es tan culta como tierna.

Y en ésta casa que tanto te se áma el Gefe que soy yo, no hai para que preguntar si estará siempre á tus órdenes_

Todo lo qué tan cariñosamente dice ése hombre inmaculado de mí, me consuela del dolor que me causa la difamacion injusta que otros hacen de mi nombre, por el hecho, del cual no soy yo responsable, de no haber nacido en ésta Tierra por cuya ventura he sacrificado, todo cuanto puede sacrificarse un hombre por la Patria agena.

Asi entiendo yo que se debe amar á Cuba. 
Leyendo á Ricardo Palma me he encontrado una vireyna, que descartando_el titulo de aquellos tiempos, es un tipo parecido á tí_Y me he dicho, "con Lola de primer Ministro sí me atrevo yo, á gobernar á Cuba".

Y allí le pregunté por ti. ;La póbre! San Francisco, como le he puesto, yo, con la diferencia que aquel fue mas afortunado porque parece que éra un buen moso que las mugeres se enamoraban de él.

A éso he llamado yo "basinilla de oro para escupir sangre".

Allá iré yo, si no mañana Sabado el Lunes.

Fernández (1999) considera que la estructura de algunas subordinadas denota énfasis. Ese podría ser el caso de las siguientes oraciones del corpus:

(...) tus cartas, rebosando afectos sinceros nos consuelan y atientan para vivir con fé, yo estimo que no tenerla es la mayor de todas las desgracias humanas. Escusandome, como tu lo sabes, sin ipocrésias (...)

No puedo contestar á tu cariñosa carta como yo deseo.

Ya hablarémos de todo éso cuando tu termines de abrasar á tus numerosos amigos.

Cuando yo sé lo qué, hombres como este y Ricardo Palma piensan de mí, tentado me siento á creer que en realidad yo valgo algo.

Ya habrá tiempo de ocuparnos de eso cuando tu vuelvas de N. York.

Cuando yo dije éso á Gilberto el Ministro, se quedó admirado.

Hasta ahora se ha intentado relacionar aquellos contextos determinados por investigaciones anteriores como enfáticos o no, con casos específicos de nuestros documentos. Sin embargo, como era de esperar, la variedad de estructuras, dentro de las que se insertan los pronombres sujetos expresos de las cartas, sobrepasa los esquemas planteados en la bibliografía consultada. Por esta razón, en adelante se tratarán aquellos ejemplos en los que no resulta evidente una clasificación.

En los textos se observan algunas construcciones en las que se despliegan las formas de primera o segunda persona del plural. En estos casos la presencia de los PPSs es necesaria para el mantenimiento de la peculiar estructura en la que se encuentran inmersos: 
Por otro lado, yo, con mi familia habíamos hecho el proposito de irnos á pasar el 10 de Oct. á Cacahual y así lo hicimos, (y así lo hicimos). (Yo + otros=Nosotros habiamos hecho el propósito...)

Manana, Clemencia y todos los mios se unen á mí para desde aquí abrasarte. Y Tu junto con Bonocio y la bella Adela reciban mi puro afecto. (Ustedes reciban)

¿Que orador "podra sacudir tu organismo en el miten" cuando tu como yo, estamos hastiados de oir palabrerias! (Nosotros estamos hastiados de oir...)

Tu que como yo ave errante apenas hemos encontrado un árbol que nos de sombra y frutas, pero que hemos sabido poner la proa de nuestra nave siempre al viento de los cíclones, (...) (Nosotros hemos encontrado...)

El cambio de referente ha sido reconocido como una variable determinativa en la explicitación de los sujetos pronominales (Orozco y Guy, 2008; Prieto, 2011; Rouges, 2011; Vidal, 2013). En la bibliografía consultada hasta el momento, no se hace alusión a ninguna posible relación entre la variable cambio de referencia y la categoría de énfasis. A continuación, se ofrece un listado de algunos de los casos de cambio de referente, que se comprueba, además, con una alta frecuencia en los textos:

Nos ocupamos de tu ojo enfermo, y, hai quien haya dicho delante de mí_! Si Lola quedara ciega"! y yo he contestado.

Como los americanos son tan buenos fabricantes en todos los ramos (mecánicos), siguen siempre, aquí como en todas [interlineado: partes], los mismos procedimientos. $\underline{\mathrm{Tu}}$ no sabes como me traen ahora á mi los mismos cubanos.

Esa noche tendremos una velada y las gentes de éstos Pueblos quieren oirte. Si puedes venir me lo dises para yo preparar, no la venida, que esa es fácil, sino el regreso que es lo penoso. Pienso que, si tu vienes, pasarémos una noche agradable.

"Para remover á un empleado es preciso andar con cuidado, pues puede condenarse una familia al hombre". Lo de la Convencion, yo creo que allá se ha de llegar pero con una mala coletilla que á mi no me ha gustado nada.

Algunos investigadores han indicado que cuando no hay cambio de sujeto gramatical $\mathrm{y}$, por tanto, se mantiene la misma referencia, los hablantes no creen necesaria la explicitación del pronombre (Orozco y Guy, 2008; Ortiz, 2009b). Sin embargo, en nuestras cartas se documentan algunos ejemplos en los que no se comprueba esta aseveración. La mayoría de estos usos pueden verse asociados a la 
lengua oral en la que, con mayor frecuencia, y como refiere Rouges (2011), el hablante siente la necesidad de reactivar el referente:

En éste asunto, como en cualquiera otro, no puedo ofrecerte mi ayuda, pues yo no puedo hacer nada en éste Mundo, sin fé, y yo la he perdido toda en mi valer é influencias.

$\underline{\mathrm{Tu}}$, Lola, has puesto tu corazon junto al nuestro en aquellas horas de pena por que tu tambien has llorado en ésta vida y con tu alma sensible no puedes pasar en tu viaje por la vida, al lado de un dolor ó una desdicha, así esté en lo alto, como arrastrandose por el suelo, sin bajarte á consolarla.

No habia podido contestar tu apreciable carta, (porqué), hijita, por falta de tiempo material De noche caigo rendido. $\underline{Y o}$ creo que ati te consta que, yo, me he tomado cuanto interes he podido por qué Fernando se coloque en otro destino mejor.

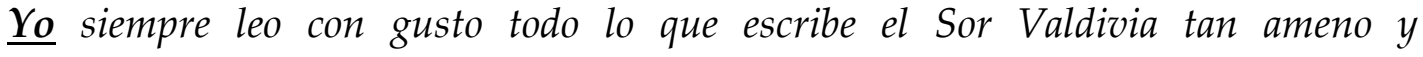
sustancioso, cualidades, que juntas no son muy comunes en escritores. $\underline{Y o}$ he contestado, así es que no debes tener quejas, tu carta dandote el parabien por el ascenso, diremos, tu hijo Fernando.

\section{DISCUSIÓN}

Con respecto a las variables analizadas como favorecedoras de la presencia de los pronombres personales sujetos de primera y segunda persona es importante aclarar que cada una de ellas podría demandar una investigación. La aproximación a este fenómeno pretendió ser un primer acercamiento a un tema de gran interés para los lingüistas y de escasos trabajos.

En este examen, de acuerdo con las variables analizadas, se comprueba que la presencia de los PPSs se ve favorecida con la primera persona del singular, el modo indicativo y el tiempo pretérito (copretérito). Según las variables de sintaxis y semántica verbal, los verbos transitivos, copulativos y de actividad cognoscitiva mental también favorecen la presencia de los PPSs. Asimismo, el cambio de referencia resulta significativo en la explicitación de estas formas pronominales, ya que muestra a la nueva persona gramatical en la que se focalizará el enunciado.

Las variables pertinentes para la ausencia de los PPSs serían, en cambio, las construcciones con formas no personales del verbo y los enunciados imperativos, ya que así lo permite el contexto.

Los textos analizados no permitieron verificar el carácter pertinente de dos de los factores que, según la bibliografía, propician la presencia de los pronombres personales sujetos: las frases interrogativas y la ambigüedad morfológica, derivada de la omisión 
fonética de la /-s/ en la flexión verbal para la segunda persona singular. Este fenómeno provocaría una indistinción entre la segunda y la tercera persona singular, por lo que motivaría la expresión del sujeto para compensar la pérdida de este fonema en la desinencia verbal y desambiguar la expresión. No documentamos en nuestras cartas la elisión de este fonema en las desinencias verbales.

Aunque la mayoría de los autores consultados aseguran que el énfasis es una de las variables más influyentes para el uso de los pronombres personales sujetos, solo pocos delimitan los contextos enfáticos de los no enfáticos. Algunos de los casos documentados en nuestro corpus no resultaron evidentes a la hora de clasificarlos teniendo en cuenta esta categoría, lo que nos puede dar la medida de que se trata de un cambio en marcha, para esta época, que podría implicar la explicitación del pronombre personal sujeto sin valor enfático. De cualquier manera, el análisis de esta categoría es una tarea que todavía espera por otros estudios lingüísticos que esclarezcan el curso de sus manifestaciones.

El sistema pronominal del español es, sin dudas, un fenómeno lingüístico complejo y vasto, por lo que solo nos limitamos a señalar, en esta primera aproximación, algunas de las variables sintácticas, semánticas y pragmáticas más relevantes que pueden propiciar el uso de los PPSs.

Los análisis aquí desplegados han de marcar el inicio de futuras investigaciones basadas en las fuentes documentales y a partir de los principios de la dialectología histórica, manera más rigurosa y confiable de hacer historia de la lengua.

\section{Aún por hacer}

Recomendamos que se promuevan estudios en diferentes contextos, zonas y con otras variables lingüísticas, por ejemplo, del tipo demosocial (edad, sexo, nivel de escolaridad) con el fin de lograr una descripción más exhaustiva del comportamiento variable de los PPSs en la variedad antillana del español.

\section{Agradecimientos}

Los autores desean agradecer a los revisores anónimos que realizaron un valioso aporte al artículo final. Además, desean agradecer a Luis Enrique Rodríguez, Maritza Carrillo, Lydia Castro, Elisabet Uría y Laura Menéndez por sus invaluables aportes en la realización de esta investigación y a la MSc. Amanda Chang Pags por su ayuda en la traducción y revisión al francés.

\section{REFERENCIAS}

Abreu, L. (2009). Spanish subject personal pronoun use by monolinguals, bilinguals and second language learners (Tesis doctoral). University of Florida, Florida, Estados Unidos. Recuperada de https://ufdc.ufl.edu/UFE0025112/00001. 
Abreu, L. (2012). Subject Pronoun Expression and "Priming Effects among Bilingual Speakers of Puerto Rican Spanish. En K. Geeslin y M. Díaz-Campos (Eds.), Selected Proceedings of the 14th Hispanic Linguistics Symposium (pp. 1-8). Recuperado de http://www.lingref.com/cpp/hls/14/paper2651.pdf.

Bentivoglio, P. (1987). Los sujetos pronominales de primera persona en el habla de Caracas. Caracas: Universidad Central de Venezuela.

Cameron, R. (1993). Ambiguous agreement, functional compensation, and nonspecific tú in the Spanish of San Juan, Puerto Rico, and Madrid, Spain. Language Variation and Change, 5(3), 305-334. doi: https://doi.org/10.1017/S0954394500001526.

Cano, R. (1981). Estructuras sintácticas transitivas en el español actual. Madrid: Gredos. Claes, J. (2011). ¿Constituyen las Antillas y el Caribe continental una sola zona dialectal?: Datos de la variable expresión del sujeto pronominal en San Juan de Puerto Rico y Barranquilla, Colombia. Spanish in Context, 8(2), 191-212. doi: 10.1075/sic.8.2.01cla.

Comesaña, S. (2002). Los verbos de conocimiento en español, caracterización sintáctica. Verba: Anuario Galego de Filoloxía, 29, 243-260. Recuperado de http://hdl.handle.net/10347/3369.

De Saeger, B. (2006). Evidencialidad y modalidad epistémica en los verbos de actitud proposicional en español. Interlingüística, (17), 268-277.

Enríquez, E. (1984). El pronombre personal sujeto en la lengua española hablada en Madrid. Madrid: Consejo Superior de Investigaciones Científicas.

Fernández, O. (1999). El pronombre personal. Formas y distribuciones. Pronombres átonos y tónicos. En I. Bosque y V. Demonte (Coords.), Gramática descriptiva de la lengua española (pp. 1209-1274). Madrid: Espasa.

Flores, N. (2004). Spanish subject personal pronoun use in New York City Puerto Ricans: Can we rest the case of English contact? Language Variation and Change, 16(1), 4973. doi: 10.1017/S0954394504161048.

Flores, N. (2005). La expresión del pronombre personal sujeto en narrativas orales de puertorriqueños de Nueva York: El español en los Estados Unidos y en contacto con otras lenguas. En L. Ortiz y M. Lacorte (Eds.), Contactos y Contextos Lingüísticos. El español en los Estados Unidos y en contacto (pp. 119-130). Berlín: Iberoamericana Vervuert.

Flores, N. (2007). Los Mexicanos in New Jersey: Pronominal Expression and Ethnolinguistic Aspects. En J. Holmquist, A. Lorenzino y L. Sayahi (Eds.), Selected Proceedings of the Third Workshop on Spanish Sociolinguistics (pp. 85-91). Recuperado de http://www.lingref.com/cpp/wss/3/paper1529.pdf.

Flores, N. (2009). Are you referring to me? The variable use of UNO and YO in oral discourse. Journal of Pragmatics, 41(9), 1810-1824. doi: 10.1016/j.pragma.2008.09.024.

Gili Gaya, S. (1975). Curso superior de sintaxis española. La Habana: Editorial Pueblo y Educación. 
Hochberg, J. (1986). Functional Compensation for /s/ Deletion in Puerto Rican Spanish. Language, 62(3), 609-621. doi: 10.2307/415480.

Hurtado, L. (2005). Condicionamientos sintáctico-semánticos de la expresión del sujeto en el español colombiano. Hispania, 88(2), 335-348. doi: 10.2307/20140954.

Kervorkian, A., y Pacagnini, A. M. (2010). Algunas observaciones acerca de los denominados 'verbos de cognición': los casos de creer y pensar + infinitivo". En V. Castel y L. Cubo de Severino (Eds.), La renovación de la palabra en el bicentenario de la Argentina. Los colores de la mirada lingüística (pp. 693-700). Mendoza: Facultad de Filosofía y Letras de la Universidad Nacional de Cuyo.

Llorach, E. (1994). Gramática de la lengua española. Madrid: Editorial Espasa-Calpe.

López, H. (1992). El español del Caribe. Madrid: Editorial MAPFRE.

López, H. (1999). Léxico disponible de Puerto Rico. Madrid: Arco Libro.

Luján, M. (1999). Expresión y omisión del pronombre personal. En I. Bosque y V. Demonte (Coords.), Gramática descriptiva de la lengua española (pp. 1275-1315). Madrid: Espasa.

Lyons, J. (1971). Introducción a la lingüística teórica (Trad. R. Cerda). Barcelona: Editorial Teide. (Obra original publicada en 1968 bajo el nombre de Introduction to Theoretical Linguistics. Cambridge: Cambridge University Press).

Martínez, J. N. (2010). Epistemicidad y subjetividad en la expresión fija creo. En V. Castel y L. Cubo de Severino (Eds.), La renovación de la palabra en el bicentenario de la Argentina. Los colores de la mirada lingüística (pp. 801-806). Mendoza: Facultad de Filosofía y Letras de la Universidad Nacional de Cuyo.

Méndez, N., y Moreno, S. (2014). La expresión de los pronombres personales sujetos en español: evidencia desde el lenguaje nativo y no nativo y sus implicaciones para la clase de Español Lengua Extranjera. En IX Conferencia Internacional sobre Lenguas Extranjeras, Comunicación y Cultura (WEFLA). Recuperado de https://www.academia.edu/6967724/La_expresi\%C3\%B3n_de_los_pronombres_per sonales_sujetos_en_espa\%C3\%B1ol_evidencia_desde_el_lenguaje_nativo_y_no_na tivo_y_sus_implicaciones_para_la_clase_de_Espa\%C3\%B1ol_Lengua_Extranjera.

Morales, A. (1997). La hipótesis funcional y la aparición de sujeto no nominal: el español de Puerto Rico. Hispania, 80(1), 153-165. doi: 10.2307/345995.

Morales, A. (1999). Anteposición de sujeto en el español del Caribe. En L. A. Ortiz (Ed.) El Caribe hispánico: perspectivas lingüísticas actuales: Homenaje a Manuel Álvarez Nazario (pp. 77-98). Frankfurt: Iberoamericana Vervuert.

Orozco, R., y Guy, G. (2008). El uso variable de los pronombres sujetos: ¿Qué pasa en la costa Caribe colombiana? En M. Westmoreland y J. Thomas (Eds.), Selected Proceedings of the 4th Workshop on Spanish Sociolinguistics (pp. 70-80). Recuperado de http://www.lingref.com/cpp/wss/4/paper1757.pdf.

Ortiz, L. A. (Ed.). (1999). El Caribe hispánico: perspectivas lingüísticas actuales: Homenaje a Manuel Álvarez Nazario. Frankfurt: Iberoamericana Vervuert. 
Ortiz, L. A. (2009a). El español del Caribe: orden de palabras a la luz de la interfaz léxico-sintáctica y sintáctico-pragmática. Revista Internacional de Lingüística Iberoamericana, 7(14), pp. 975-93. Recuperado de https://www.jstor.org/stable/41678402.

Ortiz, L. A. (2009b). Pronombres de sujeto en el español (L2 vs. L1) del Caribe. En M. Lacorte y J. Leeman (Coords.), El Español en Estados Unidos y otros contextos de contacto: sociolingüística, ideología y pedagogía (pp. 85-110). Frankfurt: Iberoamericana-Vervuert.

Otheguy, R., y Zentella, A. C. (2007). Apuntes preliminares sobre el contacto lingüístico y dialectal en el uso pronominal del español en Nueva York. En K. Potowski y R. Cameron (Eds.), Spanish in Contact: Policy, Social and Linguistic Inquiries (pp. 275295). Amsterdam: John Benjamis Publishing Company.

Pérez, M. (2006). Comportamiento de los pronombres personales en muestras de habla culta de ciudad de La Habana (Tesis de doctorado). Universidad de La Habana, La Habana, Cuba.

Posio, P. (2011). Spanish subject pronoun usage and verb semantics revisited: First and second person singular subject pronouns and focusing of attention in spoken Peninsular Spanish. Journal of Pragmatics, 43(3), 777-798. doi: 10.1016/j.pragma.2010.10.012.

Prieto, S. Y. (2011). Los pronombres personales sujetos en el español de América y España: estudio dialectal comparativo (Tesis de doctorado). Universidad de Puerto Rico, Puerto Rico.

Real Academia Española. (2006). Esbozo de una nueva gramática de la lengua española. Madrid: Espasa.

Rouges, C. (2011). El uso de los pronombres personales en función de sujeto: un estudio comparativo entre el castellano y el andaluz (Tesis de maestría). Universiteit Gent, Gante, Bélgica. Recuperada de https://lib.ugent.be/fulltxt/RUG01/001/786/602/RUG01001786602_2012_0001_AC.pdf.

Sánchez, M. (2013). Compensación funcional y sujeto pronominal "tú" en el español de Cuba. Lengua y Sociedad, 13(1), 131-154. Recuperado de http://revista.letras.unmsm.edu.pe/index.php/ls/article/view/444.

Sarmiento, R., y Esparza, M. (1994). Los pronombres. Alcobendas: Sociedad General Española de Librería.

Shinzato, R. (2004). Some observations concerning mental verbs and speech act verbs. Journal of Pragmatics, 36(5), 861-882. doi: 10.1016/S0378-2166(03)00002-X.

Silva, C. (1982). Subject expression and placement in Mexican-American Spanish. En J. Amastae y L. Elías-Olivares (Eds.), Spanish in the United States: Sociolinguistic aspects (pp.93-120). Nueva York: Cambridge University Press. 
Silva, C. (1996). Language Contact and Change: Spanish in Los Angeles. Oxford: Oxford University Press.

Suñer, M. (1986). Lexical Subjects of Infinitives in Caribbean Spanish in Studies in Romance Linguistics. En O. Jaeggli y C. Silva (Eds.), Studies in Romance Linguistics (pp. 189-203). doi: 10.1515/9783110878516.

Vandermeulen, N. (2011). El pronombre personal sujeto en el español caribeño. Un estudio comparativo entre el puertorriqueño y el venezonalo (Tesis de maestría). Universiteit Gent, Gante, Bélgica. Recuperada de https://lib.ugent.be/fulltxt/RUG01/001/786/665/RUG01001786665_2012_0001_AC.pdf.

Vaquero, M. T. (1996). El español de América. Madrid: Arco Libros.

Vidal, L. (2013). El uso variable de los pronombres sujetos en el castellano puertorriqueño hablado en Luisiana y Puerto Rico (Tesis de maestría). Louisiana State University, Louisiana, Estados Unidos. Recuperada de https://digitalcommons.lsu.edu/gradschool_theses/3876.

Villegas, C. (2006). La relación entre la posición del sujeto en el español de Venezuela con la estructura gramatical, la clase de verbo y la progresión temática: una aproximación a través de la prensa venezolana. Letras, (73), 225-274.

\section{SOBRE LOS AUTORES \\ Yesenia Ramírez Fuentes}

Licenciada en Letras, Universidad de La Habana. Estudiante de Doctorado, Pontificia Universidad Católica de Chile.

Correo electrónico: yramirez1@uc.cl

Orcid: 0000-0003-0000-3351.

\section{Damian Valdés Santiago}

Licenciado en Ciencia de la Computación, Máster en Ciencias Matemáticas, Estudiante Doctoral en Ciencias Matemáticas. Profesor Asistente y Aspirante a Investigador, Departamento de Matemática Aplicada, Facultad de Matemática y Computación, Universidad de La Habana.

Correo electrónico: dvs89cs@matcom.uh.cu

Orcid: 0000-0001-9138-9792.

\section{Marlen Aurora Domínguez Hernández}

Licenciada en Lengua y Literatura Hispánicas, Doctora en Ciencias Filológicas. Profesora Titular, Facultad de Artes y Letras, Universidad de La Habana. Miembro de la Academia Cubana de la Lengua.

Correo electrónico: marlen@fayl.uh.cu

Orcid: 0000-0001-8188-4415. 


\section{ANEXOS}

\section{Anexo 1. Transcripción de las cartas}

Para el análisis de estos documentos se llevó a cabo la tarea de transcribir y comentar cada texto. Ello permite establecer futuras comparaciones con otros documentos que estén igualmente preparados y regidos por las mismas normas de transcripción.

Tomamos como referente para la edición y transcripción de las fuentes documentales el modelo propuesto por la Red Internacional CHARTA adecuándolo a las características de la época y peculiaridades de nuestros documentos. El Corpus Hispánico y Americano en la Red: Textos Antiguos (CHARTA) fue creado en 2005 y estuvo conformado por numerosos y prestigiosos investigadores de disímiles universidades. CHARTA propone para el análisis de los textos efectuar la transcripción paleográfica de cada uno y el comentario crítico.

Los criterios de transcripción de CHARTA nos proponen una cabecera en la edición documentada, la cual es muy útil para condensar informaciones sobre la identificación del grupo investigativo, del corpus, y del documento dentro de la muestra, la procedencia del documento, data tópica y crónica, soporte y estado de conservación del documento, asunto, nombres de los transcriptores y revisores del documento.

Es conocido que en la adopción de una $u$ otra metodología investigativa subyacen, generalmente, cuestiones conflictivas que salen al paso en la práctica editorial y que encuentran provisionales soluciones a partir de convenciones adoptadas por el equipo investigativo. A continuación, se detallan algunas de las problemáticas presentadas en la transcripción paleográfica y, en consecuencia, los criterios rectores:

- No examinamos la posibilidad de brindar información sobre las indicaciones referidas a las medidas de la pieza, por lo que explica su ausencia en la cabecera del documento.

- Se decidió en la transcripción agrupar todo el contenido de la carta en un único párrafo por lo ambiguo que resulta en las misivas la delimitación de los párrafos.

- Se decidió respetar las grafías y signos de puntuación de los documentos.

- Se ha mantenido la acentuación que muestran los originales, tanto en lo que se refiere a su ausencia o presencia.

- Los subrayados en el texto original se mantienen: puedo, quiero, todo.

- No se desarrollan las abreviaturas ni las letras voladas, se mantienen como tales: qe, pa, Eno. 
- Las palabras que han sido separadas en sílabas en los textos y presenten el guión de separación, serán representadas sin espacios en blancos entre la primera parte de la palabra, el guion, el símbolo correspondiente a la línea y la segunda parte de la palabra, como se muestra a continuación, so-\{5\}bre.

- Se respetan las amalgamas con pronombres y determinantes, como meha, mehicieron.

- En la transcripción se nos presentó como duda si debíamos tomar la despedida y la firma individualmente como una línea. Finalmente, se decidió fijar como línea solamente la despedida y a continuación, entre corchetes, la firma del remitente.

- Con respecto a la fecha también se concluyó que esta aparecería como una línea, estuviera al inicio o al final del texto.

- El saludo también se contemplaría como línea.

A modo de ejemplo se ilustra lo antes señalado en una de las cartas:

$1 A$

HISDELE

1900 julio $5<<$ s.l. $>>$

Se trata de una carta personal, donde el destinador, Máximo Gómez, le comunica a su destinataria, Lola Rodríguez de Tió su deseo de verla con prontitud para tratar un asunto que le interesa, por lo que exige la urgente confirmación de su destinataria para visitarla.

El tipo de soporte es papel.

La pieza se conserva en buen estado.

Transcriptor (a): Yesenia Ramírez Fuentes

1er.Revisor: Dra. Marlen A. Dominguez Hernández $\{h$ 1r\} \{1\} Señora Lola d.Tió \{2\} Mi estimada Lola $\{3\}$ tengo necesidad $\{4\}$ de que hablémos so-\{5\}bre un asunto, pero \{6\} sin testigo, que me $\{7\}$ interesa $\{8\}$ Dime si puedo $\{9\}$ ir ésta tarde á las $\{10\} 4$ á tu casa. \{11\} Queredor amigo [firma: M. Gomez] [signo] \{12\} 5 Julio 1900 